\title{
La motivación y el uso de estrategias en la enseñanza - aprendizaje para docentes del idioma inglés
}

Motivation and the use of teaching strategies - learning for English language teachers

Rocío de los Ángeles Barragán Murillo. ${ }^{1}$, Esthela Isabel Colcha Guashpa. ${ }^{2}$, Zoila Victoria Herrera Andrade. ${ }^{3}$

\section{Recibido: 22-04-2019 / Revisado: 26-05-2019 /Aceptado: 27-06-2019/ Publicado: 30-07-2019}

\begin{abstract}
DOI: _https://doi.org/10.33262/cienciadigital.v3i3.2.1.857

Motivation plays a very important role in achieving a successful development of English classes. It has become a pedagogical need whose objective is to expose ways that motivate the English teachers to do their best in class, so that motivation can be used as a pivotal means in the design of stimulating classes based on the reality of their students; that is why the use of techniques that contribute to raising the degree of interest in the area of teaching learning by encouraging a positive teachers' attitude when teaching classes, therefore the attitude of students towards the language.
\end{abstract}

Keywords: Motivation, Teachers, English, Students, Positive Attitude, Strategies.

\section{Resumen}

La motivación juega un papel de mucha importancia para lograr el desarrollo exitoso de las clases de inglés. Convirtiéndose en una necesidad pedagógica cuyo objetivo es exponer vías que motiven al docente de inglés al mejor ejercicio de su labor educativa, para que este a su vez utilice esa motivación como medio ineludible en el diseño de sus horas de clases estimuladoras basadas en la realidad de sus estudiantes. Por lo que el uso de técnicas fomente y contribuyan a elevar el grado de interés por lo novedoso en el área de enseñanza aprendizaje causando una actitud positiva de los docentes al momento de impartir las clases, por ende, la actitud de los estudiantes frente al idioma inglés.

Palabras Claves: Motivación, Docente, Inglés, Estudiantes, Actitud Positiva, Estrategias.

\section{Introducción}

El inglés en la época actual es la lengua de habla en todo el mundo; de aquí se deduce la importancia del aprendizaje de este idioma en los estudiantes. En la gran mayoría de países del mundo, el inglés se imparte dentro del sistema educativo, desde la educación inicial hasta la formación profesional. Sin embargo, resulta preocupante que, al finalizar

\footnotetext{
${ }^{1}$ Escuela Superior Politécnica de Chimborazo, Chimborazo, Ecuador, robarragan@espoch.edu.ec

${ }^{2}$ Escuela Superior Politécnica de Chimborazo, Chimborazo, Ecuador, e_colcha@espoch.edu.ec

${ }^{3}$ Escuela Superior Politécnica de Chimborazo, Chimborazo, Ecuador, zoila.herrera@espoch.edu.ec
} 
el bachillerato, los estudiantes poseen conocimientos muy elementales acerca del idioma, resultando insuficiente al ingresar al nivel de educación superior.

Se puede considerar las posibles causas de esta situación, en su mayoría coinciden en la importancia que tiene la motivación de los jóvenes para hacer más positivo el proceso de enseñanza aprendizaje del inglés. Se ha observado a través del tiempo la existencia de numerosos trabajos de investigación, que precisan lo primordial del aspecto motivacional para la implementación de las clases de otros idiomas como el inglés, dando la responsabilidad absoluta al docente, quien tiene la tarea de hacer de la motivación su herramienta diaria en pro de favorecer el desarrollo de cada clase.

Sin embargo, nos preguntamos qué pasa cuando la realidad difiere en gran medida de lo esperado. Se puede decir que se habla de la motivación del estudiante sin tomar en cuenta la motivación de quién también se debe motivar; es decir el docente. Quien es el encargado de planificar clases divertidas y pertinentes a cada tema, adaptadas a las necesidades de sus estudiantes, creando una atmosfera lo suficientemente agradable para que las jornadas de trabajo se desarrollen de manera favorable. Por ello consideramos que un docente motivado es igual a los alumnos motivados.

La importancia radica en que los docentes de inglés, demuestren su desempeño dentro del aula y se abran a nuevas formas de acercamiento al idioma, expresen sus necesidades en el área e identifiquen sus posibles limitaciones, así como también puedan compartir con otros profesionales las experiencias con las cuales les permita enriquecer su formación en la enseñanza aprendizaje de esta lengua.

\section{Motivación}

Según el autor Abraham Maslow psicólogo norteamericano, la motivación es el impulso que tiene el ser humano de satisfacer sus necesidades. Maslow clasifica estas necesidades en 5 y las clasifica en una pirámide las cuales en su base ubica a la fisiología, seguridad, afiliación, reconocimiento y autorrealización.

(Sanfeliciano, Alejandro., 2018) La motivación en la educación es uno de los aspectos esenciales que se deben tener en cuenta. Un sistema educativo que ayude a los alumnos a afrontar las tareas y a cumplir sus retos es necesario para lograr un aprendizaje de calidad. Para ello, hay que hacer un análisis exhaustivo sobre estos aspectos motivacionales.

Motivación, según Valenzuela González (1999), es el conjunto de estados y procesos internos de la persona que despiertan, dirigen y sostienen una actividad determinada. Concepto que involucra a que el estudiante este motivado y pueda a través de ello estimular su actividad convirtiendo su ánimo por estudiar en una disciplina de estudio en base al logro de objetivos de aprendizaje para una alcanzar su satisfacción.

\section{Tipos de motivación.}

\section{Se ha tomado esta}

Motivación relacionada con la tarea, o intrínseca: El autor Francisco Sáez (2019), manifiesta que la motivación intrínseca es la que nos impulsa a hacer cosas por el simple gusto de hacerlas. La propia ejecución de la tarea es la recompensa. A diferencia de la motivación extrínseca la cual se basa en obtener dinero, recompensas y castigos, o presiones externas, la motivación intrínseca nace en el propio individuo. Con lo cual se refiere a que se despierta el interés en el estudiante de la materia que se está estudiando 
generando satisfacción en el estudiante mientras empieza a alcanzar el objetivo de estudio.

Motivación relacionada con el yo, con la autoestima: Al intentar aprender y conseguirlo vamos formándonos una idea positiva de nosotros mismos, que nos ayudará a continuar con nuestros aprendizajes. Las experiencias que tienen los alumnos van formando poco a poco el auto concepto y la autoestima. Es el deseo constante de superación, guiado siempre por un espíritu positivo. (Conde, 2010). Este pensamiento enfatiza en el conocimiento interno que cada alumno tiene de sí mismo y el potencial que posee para alcanzar sus metas propuestas.

Motivación centrada en la valoración social: La aceptación y aprobación que se recibe por parte de las personas que el alumno considera superiores a él. La motivación social manifiesta en parte una relación de dependencia hacia esas personas. (Rochina, 2012). Esto se refiere al reconocimiento que espera el estudiante recibir de sus superiores asintiendo que lo realizado por él en su aprendizaje sea reconocido por sus maestros.

Motivación que apunta al logro de recompensas externas: En este caso estamos hablando de los premios, regalos que se reciben cuando se han conseguido los resultados esperados. (La motivación en el aprendizaje motor., 2014). En este aspecto se refiere a los estímulos materiales a los que se hace acreedor el estudiante cuando logra un fin propuesto, con lo cual su aceptación y reconocimiento lo engrandece.

\section{Enfoques}

El Método basado en la Gramática y en la Traducción: (Mackey, 1965. Pág. 153) expresa: es un método deductivo y mentalista, según el cual el idioma se adquiere aprendiendo memorísticamente las reglas y paradigmas gramaticales, y largas listas de vocabulario, y se practica aplicando esos conocimientos en ejercicios de traducción directa e inversa. El uso de este método permite un aprendizaje memorístico para la asimilación de información mediante la aplicación de ejercicios que implican la traducción.

El Método Directo se da cuando el aprendizaje de una lengua extranjera se planteó como objetivo la comunicación con los hablantes de esa lengua, y no sólo la lectura de obras literarias, se vio que el método basado en la gramática y la traducción no podía seguir utilizándose, o al menos no de forma exclusiva. Surgen así, en las décadas de los años veinte y treinta, una serie de iniciativas metodológicas, posteriormente agrupadas bajo la denominación de "métodos directos", cuyo denominador común es la idea de que al estudiante hay que ponerlo en contacto directo con el idioma que quiere aprender, simulando situaciones concretas lo más parecidas a las reales que se pudieran conseguir en el aula. (Jespersen, 1922). Este método permite un aprendizaje inductivo, mediante la comunicación oral.

El método estructuralista o audio-lingual, Sobre este método Hernández Reinoso (2000), manifiesta que este método tiene como base el supuesto de que el aprendizaje de una lengua debe realizarse a partir de la internalización de hábitos lingüísticos y no a partir de la memorización de reglas (p.103-buscar). Por otra parte, busca que los estudiantes aprendan nuevo vocabulario mediante la asociación de palabras habladas e imágenes visuales, se enfatizan los ejercicios repetitivos e imitación de patrones nativos y para esto recurren a grabadoras y otros medios tecnológicos. Se deduce que el uso de 
este método permite un aprendizaje mediante, la escucha y la repetición para producir en el estudiante una interiorización del idioma en sí.

El enfoque cognoscitivo, básicamente se encarga del estudio de los procesos mentales implicados en el conocimiento. A diferencia del conductismo, en el cognoscitivismo, los estímulos no son determinantes directos de la conducta, es decir el aprendizaje, si no, los procesos internos por los cuales el sujeto procesa esos estímulos. El término cognoscitivo hace referencia a actividades intelectuales internas como la percepción e interpretación del pensamiento. Este enfoque es muy importante ya que conduce a nuevas ideas sobre la representación y naturaleza del conocimiento y también hacia algunos fenómenos como la memoria, la solución de problemas, el significado que se le da a las cosas, la comprensión y la producción. (Diaz, Luis G. , 2018). Ante lo expuesto podemos decir que este enfoque se encarga del conocimiento mediante los estímulos que el estudiante recibe para emitir nuevas ideas y soluciones a toda la información que se le presente.

El enfoque comunicativo surgió en los años 70 como consecuencia de un cambió en la perspectiva de la enseñanza de las segundas lenguas. Se abandonó el modelo estructuralista para centrarse en la idea que de la lengua no es solo un objeto de conocimiento, sino ante todo un instrumento de comunicación. A partir de ahí se fundamentó la idea de que conocer una lengua no supone conocer las normas que rigen el proceso comunicativo, sino ser capaz de utilizar cualquier recurso o estrategia que sirva para facilitar y construir el acto de comunicación. Se pone el énfasis no solo en lo correcto gramaticalmente sino también en la adecuación de los enunciados a la situación y al contexto comunicativo, porque saber hablar una lengua no es sino saber qué decir y cómo decirlo en cada contexto o situación (García Armendáriz, M. V., Martínez Mongay, A. M., \& Matellanes Marcos, C.. , 2003). El empleo de este método lo hace flexible al permitir utilizar cualquier recurso o estrategia para construir la comunicación efectiva del idioma extranjero.

\section{Estrategias de enseñanza}

La autora Yolanda Campos (2000), en su documento Estrategias de enseñanza aprendizaje, expresa que la estrategia de enseñanza se refiere al arte de proyectar y dirigir; el estratega proyecta, ordena y dirige las operaciones para lograr los objetivos propuestos. Así, las estrategias de aprendizaje hacen referencia a una serie de operaciones cognitivas que el estudiante lleva a cabo para organizar, integrar y elaborar información y pueden entenderse como procesos o secuencias de actividades que sirven de base a la realización de tareas intelectuales y que se eligen con el propósito de facilitar la construcción, permanencia y transferencia de la información o conocimientos. Específicamente, las estrategias sirven para el cumplimiento de los objetivos y la obtención de la información. Las estrategias de aprendizaje se entienden como un conjunto interrelacionado de funciones y recursos, capaces de generar esquemas de acción que hacen posible que el alumno se enfrente de una manera más eficaz a situaciones generales y específicas de su aprendizaje; que le permiten incorporar y organizar selectivamente la nueva información para solucionar problemas de diverso orden. El alumno, al dominar estas estrategias, organiza y dirige su propio proceso de aprendizaje (González, 2001). Concretamente la autora se refiere a que este procedimiento ayuda al estudiante a ser más eficiente en la selección de información y en la solución de problemas que se pudieren presentar. 
El empleo de estrategias de enseñanza permite que este proceso se dé entre docente y estudiante, siendo un vínculo único. Entendido esto podemos decir que es difícil pensar que existe una sola forma de impartir el conocimiento y su aplicación sea efectiva para el aprendizaje de los estudiantes.

El autor Willian Peralta (2016) expone en su artículo Estrategias de enseñanza aprendizaje del inglés como lengua extranjera, lo siguiente:

De acuerdo con Rubín Joan y Chamot U. Anna (1985), Las estrategias de aprendizaje en una lengua extranjera se consideran un conjunto de pasos, rutinas, planes que utilizan los estudiantes con el propósito de facilitar la obtención, recuperación, almacenamiento y uso de una lengua extranjera. Estos autores consideran que las estrategias de aprendizaje de una lengua extranjera se dividen en estrategias cognitivas, metacognitivas y socio-afectivas.

Dentro de este grupo de estrategias cognitivas con el proceso de aprender una lengua extranjera, se pueden mencionar las siguientes:

- Clasificación y verificación: Se utilizan para verificar la comprensión de la lengua.

- Predicción, inferencia inductiva: En esta estrategia el educando tiene que hacer uso de su lengua materna para poder inferir el significado, es así que debe hacer uso de los conocimientos previos tanto lingüísticos como conceptuales.

- Razonamiento Deductivo: En esta estrategia el estudiante usa un patrón general para resolver su problema de aprendizaje, en el cual se debe hacer uso de analogías, síntesis.

- Práctica: en esta el punto principal en el estudiante es la exactitud en la lengua, a través de ensayo, error, repetición, imitación.

- Memorización: Lo fundamental de esta estrategia es que el estudiante retenga la información.

- Monitoreo: el estudiante tiene la capacidad de dar seguimiento e identificar las partes fuertes y débiles en su aprendizaje.

- Contextualización: en esta estrategia el estudiante busca por sí mismo dar sentido a la frase que desea aprender a manera de secuencia.

- Toma de nota: con este tipo de estrategia el estudiante busca llevar a cabo un control de las ideas principales o informaciones que el /ella considera necesario para aprender.

Las estrategias meta cognitivas: le permiten al alumno guiar o regular su propio proceso de aprendizaje. Dentro de este grupo o subclasificación podemos encontrar.

- Organizadores previos: ocurre cuando se hace por anticipado una revisión del material por aprender.

- La atención dirigida: cuando se decide atender a determinada tarea o material dentro de un contenido general.

- Atención selectiva: ocurre cuando el alumno decide retener o atender a detalles específicos sobre determinado material con el fin de retener un material específico. 
- Autoadministración: cuando el alumno determina identificar las condiciones de aprendizaje que le permitan aprender.

- Autoevaluación; con ella el alumno busca identificar utilizando sus propios métodos que tantos avances ha obtenido en determinado lapso de tiempo.

Las estrategias socio-afectivas; le permiten al individuo practicar la lengua e intercambiar información. Esta se puede presentar en dos grupos.

- Cooperación; cuando trabaja junto a otros para obtener retroalimentación.

- Aclarar dudas; aquí el alumno ignora algún detalle, pero es claro en buscar la información concreta sobre determinado contenido.

\section{Docentes}

Suárez (1991), sostiene que el educador deja de ser un transmisor de conocimientos y se convierte en un facilitador de experiencias que planifica el proceso de enseñanza, motiva, refuerza y colabora con los estudiantes, plantea problemas, genera discusiones y es capaz de evaluar a sus estudiantes y a la vez evaluarse a sí mismo.

(Dewey, 1938) afirmaba que las materias no se hacían interesantes decorándolas o aderezándolas con detalles irrelevantes. Para que una materia sea interesante, hay que realizar una instrucción que permita a los alumnos entender su complejidad, ya que el simple hecho de comprender algo es atractivo para cualquier ser humano. El problema nace cuando la instrucción no es adecuada y el alumno no comprende la materia. De esta forma, los datos que aprende carecen de significado y de interés alguno.

\section{Importancia de la formación docente en la actualidad}

Actualmente la educación ha sufrido transformaciones y reformas en sus planes y programas, siendo más exigentes para adentrarse a la nueva sociedad del conocimiento y donde precisamente la educación exige estudiantes con competencias sólidas para su ejecución. En este sentido, las escuelas de hoy no sólo preparan para culminar un nivel educativo, sino además deben de preparar a sus estudiantes para el futuro en el que se van a enfrentar de manera que, comprendan los problemas actuales desde una perspectiva objetiva, implementando las competencias adquiridas, por ello, los docentes deben estar actualizándose en todos los ámbitos con la finalidad de cumplir los perfiles de egreso que se exigen. (Díaz Barriga Arceo, 2002)

Es preciso que los docentes de hoy en día sean conscientes de que son parte de una sociedad denominada sociedad del conocimiento que requiere que los estudiantes desarrollen competencias para que las clases y las actividades sean desarrolladas de mejor manera, y así se logre cambios que sean precisos. Los docentes son ejemplos a ejemplo a seguir. En tal virtud, es necesario crear ambientes de aprendizajes significativos augura un involucramiento y desarrollo de competencias dentro y fuera de la escuela. (Alviárez, 2005)

Arredondo (1989) describe que el docente además de ser facilitador también funge el papel de investigador, asesor y guía, señalando que debe indagar respectos de los intereses de los alumnos, el contexto cultural, social y escolar, factores importantes que dicha investigación o reconocimiento permitirá saber que estrategias y/o técnicas se deben aplicar, logrando así que los alumnos cumplan con los aprendizajes esperados. 


\section{Rol del docente de ingles}

El rol del docente se basa en ser un guía para sus estudiantes en el proceso de aprendizaje de la lengua extranjera, brindando herramientas para desarrollar de manera adecuada sus habilidades". (Gonzales, 2019)

El docente de inglés ha estado sujeto a cambios a medida que la educación ha ido avanzando motivo por el cual el educador tiene que estar en constante capacitación, adquisición y actualización de conocimientos en las metodologías de enseñanza aprendizaje y herramientas tecnológicas que ayuden al buen desempeño académico.

La implementación de actividades en la educación del siglo XXI juega un papel fundamental ya que el estudiante tiene un rol activo dentro de las clases y no solo se limita a estar sentado o escuchar lo que dice el docente ya que en la actualidad existen recursos tecnológicos que permiten el aprendizaje de forma más dinámica, es decir consiste en actividades que permite desarrollar con más efectividad las destrezas del idioma inglés además de mejorar la práctica docente y motivar a los estudiantes al aprendizaje de la lengua extranjera mediante uso de técnicas existentes en la educación actual. (Aleman, Franco. y Mora, Fátima., 2017)

Se requiere de un docente con mucho tiempo extra para preparar las clases y anticiparse a las posibles dudas de vocabulario relacionadas a la disciplina a través de la cual se imparte la asignatura Se necesita un docente con extrema autoconfianza y sin temor de encarar un gran compromiso frente a una clase que aspira que sus dudas, tanto lingüísticas como teóricas, sean disipadas de forma exitosa. (Hernandez, 2000)

\section{Inglés}

El inglés, una lengua surgida dentro del territorio del Reino Anglosajón de Inglaterra, tiene un origen germánico occidental. Se considera una de las lenguas con más influencia dentro del mundo actual, siendo utilizada para dar discursos que reúnen individuos de naciones con idiomas totalmente diferentes, es decir, es una lengua franca, debido a la amplia cantidad de hablantes que posee (alrededor de 508 millones). Como tal, el término “inglés" es una derivación de "anglos", el nombre oficial de la tribu germánica que llevó un primitivo inglés a lo que se convertiría en el Reino Unido. Sin embargo, esta palabra también se puede referir al gentilicio de Inglaterra. (Conceptodefinicion.de, 2019)

El idioma inglés es considerado a nivel mundial en la actualidad como el más importante en razón de que se ha convertido en un idioma universal, puesto que las personas en su mayoría lo hablan como segunda lengua.

El dominio que debe tener el docente de una segunda lengua es crucial para la enseñanza aprendizaje de sus estudiantes para impartirlo en clases, permitiendo a los mismos dotarse de destrezas comunicativas que amplían sus horizontes hacia otras cultura y modos de vida.

\section{Estudiantes}

Los autores Santiana y Maldonado ( 2015), manifiestan en su documento:

“" El estudiante, por su parte, se convierte en un sujeto activo dentro del proceso educativo; un ser que piensa, crea, transforma, se informa, consulta, critica, discute, organiza y planifica su propio aprendizaje y es capaz de autoevaluarse. 
Dentro de esta nueva concepción, el profesor ya no será el único que aporta información, sino que el estudiante también puede enriquecerlo aportando sus propios conocimientos e ideas, para que el aprendizaje sea más efectivo y significativo. De esta manera, la función del educador será orientar y guiar explícita y deliberadamente todas las actividades de los estudiantes y crear las condiciones óptimas para que se produzca el aprendizaje"'”. Las transformaciones en el campo educativo también tuvieron repercusiones en la enseñanza específica de lenguas extranjeras, especialmente en la enseñanza del inglés; este idioma comienza a tomar importancia a nivel mundial por ser el lenguaje de la ciencia y la tecnología. De allí, según O"Dwyer, (2009)”, el viejo ideal pedagógico del profesor como autoridad que transmite conocimientos a los estudiantes es sustituido por un enfoque más democrático, centrado en el estudiante, en el cual el profesor facilita actividades educativas y comunicativas a los estudiantes. Pág.

16.

Malavé (1996), manifiesta que la enseñanza de un segundo idioma requiere el desarrollo de actividades basadas en experiencias concretas que activen los esquemas apropiado para incorporar el nuevo conocimiento del idioma, así como de las materias de estudio para promulgar tanto la adquisición del segundo idioma como el aprendizaje de conceptos de ciencia. En otras palabras, este enfoque multidisciplinario requiere la integración sistemática de las actividades para la enseñanza del inglés como segundo idioma con actividades concretas para enseñar ciencia, o ambas y en donde los estudiantes usen materiales manipulativos.

\section{Realidad del estudiante universitario}

El enseñar a estudiantes de pregrado es una tarea compleja, porque su dominio de inglés es escaso o nulo, motivo de preocupación para los docentes encargados de esta actividad. Primero porque los estudiantes poseen muy poco conocimiento lingüístico acerca de su propio idioma español. A este respecto Hidalgo (2001) enfatiza lo siguiente: Se ha observado que algunos estudiantes universitarios parecen no haber adquirido su lengua materna, pues presentan dificultad para comprenderla y expresarse a través de ella. Este problema se hace más notorio cuando la carrera que se estudia implica el aprendizaje de una segunda lengua. (p.1) 
Con el propósito de nivelar esta debilidad los profesores de inglés con propósitos específicos primero abordan gramaticalmente el idioma español, explicando los rasgos más característicos que lo componen, para luego hacer el enlace o transición hacia el idioma inglés. De no existir esta deficiencia en los estudiantes, sería mucho más fácil introducir la terminología técnica relacionada con aspectos lingüísticos del idioma antes mencionado. Toda esta situación genera un consumo de tiempo importante en la planificación de actividades durante los lapsos académicos, que muy bien podrían aprovecharse para reforzar el dominio en otras áreas donde los participantes tengan otras desventajas. (Hidalgo, 2001)

Segundo en su mayoría son estudiantes cuyo conocimiento de este idioma extranjero se limita a lo aprendido durante el bachillerato, y lamentablemente como es bien sabido, su preparación no es la más adecuada. Hernández (2001) resalta la deficiente preparación que tienen del idioma inglés los estudiantes de bachillerato. Esta autora señala que este bajo dominio se debe básicamente a las pocas horas estipuladas para esta asignatura durante cada año académico, carencia de materiales didácticos pertinentes al área, así como también limitadas y obsoletas estrategias educativas adaptadas al alto número de participantes por sección y a la falta de recursos.

\section{Actitud positiva}

De acuerdo a Brown (2000) las actitudes son cognitivas y afectivas. Estas tienen estrecha relación con los pensamientos, sentimientos y emociones. Las actitudes rigen la manera en que las personas se relacionan con los procesos de aprendizaje, que, en el caso del idioma, requiere no sólo exponerse a una lengua distinta, sino también a una cultura. Esto se refiere a que el conocimiento y sentimiento permite una mejor interacción hacia el aprendizaje.

Las actitudes comienzan a desarrollarse desde muy temprano y son influenciadas por muchos factores, incluyendo la familia, los amigos e interacciones sociales con individuos de otras culturas y costumbres. Por lo tanto, las actitudes forman parte del bagaje perceptivo que posee una persona de sí misma, de otros, y del ambiente cultural que este habita. (Díaz Alcauza, 2018)

Las actitudes positivas y negativas se ven reforzadas por las experiencias vividas por los aprendices, siendo la motivación uno de los aspectos más importantes para que el estudiante presente una buena actitud para el aprendizaje y dominio de un segundo idioma, como es el inglés.

$\mathrm{Al}$ experimentar una actitud positiva en la vida de los estudiantes adquiriendo éxito en cualquier actividad que realizan automáticamente estas se fortalecen, por el contrario, si poseen los estudiantes una actitud negativa estarán orientados al fracaso, tendiendo a volverse más rehaceos a progresar. Por lo tanto, el aprendizaje en si no es meramente un argumento de inteligencia y aptitud sino de actitud y motivación.

Brown (1980) establece que el proceso de aprendizaje de un segundo idioma necesariamente debe involucrar un completo compromiso por parte del estudiante, y por ello se entiende: interés físico, intelectual y emocional, necesarios para satisfactoriamente enviar y recibir mensajes lingüísticos. Si estas condiciones no están dadas, difícilmente tanto el estudiante como el docente lograrán la meta deseada. 
Gardner (1985) explica que las actitudes son un componente fundamental de la motivación la cual, en este contexto, representa "una combinación del esfuerzo y del deseo de querer lograr una meta educativa en contraste con la actitud que se tiene en torno al aprendizaje de una lengua extranjera".

Deci y Ryan (1985) clasifican a la motivación como intrínseca y extrínseca. Los estudiantes están intrínsecamente motivados cuando se muestran interesados en realizar actividades que se ven reflejadas en la superación de los obstáculos, despertando así sentimientos de autorrealización y autodeterminación que se traducen en el manejo de destrezas y competencias específicas.

Si bien es cierto los estudiantes se sienten motivados hacia el aprendizaje, y para el logro del mismo llevan a cabo actividades diseñadas y acompañadas por una disposición positiva hacia el aprendizaje, la misma que se traduce en un mayor esfuerzo y trabajo, obteniendo mejores resultados en el aprendizaje de una lengua extranjera.

\section{Conclusiones}

En conclusión, la importancia de contar con estrategias para la enseñanza aprendizaje del idioma ingles permiten al docente analizar lo que no son favorables y considerar aquellos más viables para hacer una clase dinámica permitiendo asimilar la gramática y el vocabulario, así como la comunicación con el estudiante.

El docente es el actor fundamental en la clase quien está presto a apoyar y guiar en la producción y modo de responder a las inquietudes que se presentan en el aula de clases para que sus estudiantes alcancen sus metas propuestas mediante la motivación y el contexto social.

Tanto docentes como estudiantes deben mantener siempre una actitud positiva y disfrutar del aprendizaje del día a día, en especial si se trata de un nuevo idioma el cual va a beneficiar y generar grandes satisfacciones al conceder oportunidades en el ámbito personal y laboral.

$\mathrm{Al}$ analizar las diferentes definiciones expuestas en este documento, se puede decir que tememos una amplia información para poder posiblemente aplicar la mejor estrategia de enseñanza y llevar a los estudiantes a alanzar un aprendizaje significativo.

\section{Referencias}

Acosta, Joel., Escobar Magda.,\& Maita, Nancy. 2011. Propuesta de estrategias de enseñanza aprendizaje para docentes de ingles. Caracas : Instituto Pedagogico de Caracas, 2011.

Aleman, Franco. y Mora, Fátima. 2017. dspace.utb.edu.ec. [En línea] 2017. [Citado el: 17 de 09 de 2019.] http://dspace.utb.edu.ec/handle/49000/3120.

Alviárez, L. 2005. "El uso de estrategias constructivistas por docentes de Inglés con Fines Específicos". 2005.

Arredondo, M. 1989. Notas para un modelo de docencia. Formación pedagógica de profesores. Mexico : ANUIES-UNAM. CESU, 1989.

Brown, H.D. (2000) . Principles of language. NY, USA: : Addison Wesley Longman. , (2000) .

Campos, Yolanda. 2000. Estrategias de enseñanza-aprendizaje". Mexico : s.n., 2000. 
Conceptodefinicion.de. 2019. conceptodefinicion.de. [En línea] 25 de 07 de 2019. [Citado el: 19 de 09 de 2019.] https://conceptodefinicion.de/ingles/. Consultado el 19 de septiembre del 2019.

Conde, Cristina. 2010. /www.pedagogia.es. [En línea] 04 de 12 de 2010. [Citado el: 19 de 09 de 2019.] https://www.pedagogia.es/motivacion-tipos-motivacion-motivar-alumno/.

Deci, E. L., y Ryan R. M. 1985. Intrinsic Motivation and Self-determination in Human Behaviour. N.Y, USA : Plenum, 1985.

Dewey, John. 1938. Experiencia y Educacion. España : Casa Blanca, 1938.

Díaz Alcauza, Cristina. 2018. Iondonacademyonline.com. [En línea] 18 de 04 de 2018. [Citado el: 18 de 09 de 2019.] http://www.londonacademyonline.com/la-importancia-unaactitud-positiva-frente-al-aprendizaje-idioma/.

Díaz Barriga Arceo, Frida. 2002. Estrategias docentes para un aprendizaje significativo una interpretación constructivista. México. : Editorial McGraw-Hill Interamericana Editores, S. A. de C. V.,, 2002.

Diaz, Luis G. . 2018. asomateunivo.com. [En línea] 15 de 04 de 2018. [Citado el: 17 de 09 de 2019.] http://asomateunivo.com/articulo/id/2913. Fundamentos Cognoscitivos: $L a$ Enseñanza del Inglés Como. Malavé, Lilliam. 1996. 11, s.I. : NYSABE JOURNAL, 1996.

García Armendáriz, M. V., Martínez Mongay, A. M., \& Matellanes Marcos, C.. . 2003. Español como segunda lengua (E/L2) para alumnos inmigrantes. Gobierno de Navarra : Pamplona, 2003. http://dpto.educacion.navarra.es/publicaciones/pdf/espanolel2.pdf.

Gardner, R.C. 1985. Social Psychology and second language learning. The role of attitudes and motivation. . London : s.n., 1985.

Gonzales, Heriberto. 2019. occidente.co. [En línea] 17 de 09 de 2019. [Citado el: 17 de 09 de 2019.] https://occidente.co/opinion/cibernautas/el-rol-de-profesor-de-ingles-comoidioma-extranjero/.

González, Virginia. 2001. Estrategias de enseñanza y aprendizaje. 2001.

Harris, D.P. (. 1969. Testing English as a second language. New York : McGraw-Hill, 1969.

Henson T., Kenneth. 2000. Psicología educativa para la enseñanza eficaz. s.I. : Editorial Internacional Thomson Editores, S. A. de C. V, 2000.

Hernández, M. 2001. Aprendizaje Cooperativo y Rendimiento Académico en Inglés en la Tercera Etapa de Educación Básica. . Trabajo de Grado de Maestría, : UC, 2001.

Hernandez, Reinoso. 2000. El metodo audio lingual. 2000.

Hidalgo, H. 2001. Estructuras Gramaticales de Adquisición Tardía en Castellano y Desarrollo Cognitivo. Trabajo de Grado de Maestría, UC, 2001 . 2001.

Jespersen, Otto. 1922. Language, its nature, Development and Origin . Roskilde : s.n., 1922. La motivación en el aprendizaje motor. 
Villegas, Fernando \& Martínez, Miguel A. 2014. 142, Buenos Aires : http://www.efdeportes.com/, 2014, Vol. Año 2014.

Mackey. 1965. Pág. 153. Los enfoques de la motivacion . México : Mc Graw Hill, 1965. Pág. 153.

Maslow, Abraham. 1943. Teoría sobre la motivación humana . 1943.

Not, Luis. 1983. Las pedagogías del conocimiento. . México : Ed. Fondo de Cultura Económico, 1983.

Peralta, Wilian M. 2016. vinculando.org/educacion/estrategias-ensenanza-aprendizaje-delingles-lengua-extranjera.html. [En línea] 2016. [Citado el: 19 de 09 de 2019.] http://vinculando.org/educacion/estrategias-ensenanza-aprendizaje-del-ingles-lenguaextranjera.html.

Rochina, Maria. 2012. mariajosefarochinagarcia.blogspot.com. [En línea] 04 de 2012. [Citado el: 19 de 09 de 2019.] http://mariajosefarochinagarcia.blogspot.com/2012/04/centrada.html.

Sáez, Francisco. 2019. facilethings.com/. [En línea] 2019. [Citado el: 19 de 09 de 2019.] https://facilethings.com/blog/es/intrinsic-motivation.

Sanfeliciano, Alejandro. 2018. lamenteesmaravillosa.com. [En línea] 8 de 05 de 2018. [Citado el: 17 de 09 de 2019.] https://lamenteesmaravillosa.com/la-motivacion-en-la-educacion/ .

Santiana Yunapanta Liliam Maritza \& Maldonado Ochoa María Luisa . 2015. repositorio.ug.edu.ec. [En línea] 05 de 2015. [Citado el: 18 de 09 de 2019.] http://repositorio.ug.edu.ec/bitstream/redug/12947/1/Maldonado\%20-\%20Santiana.pdf.

Smart. 2016. smart.edu.co. [En línea] 22 de 09 de 2016. [Citado el: 17 de 09 de 2019.] https://smart.edu.co/academia-de-ingles-2/.

Valenzula Gonzalez, J.R. 1999:16. La motivación en la educacion a distancia. En: Actas Jornadas de Informática Educativa. Buenos Aires, : s.n., 1999:16. Vol. XV.

\section{\Ciencia}




\section{PARA CITAR EL ARTÍCULO INDEXADO.}

Barragán Murillo, R., Colcha Guashpa, E., \& Herrera Andrade, Z. (2019). La motivación y el uso de estrategias en la enseñanza - aprendizaje para docentes del idioma inglés. Ciencia Digital, 3(3.2.1), 310-322.

https://doi.org/10.33262/cienciadigital.v3i3.2.1.857

\section{Ciencia \\ LDigital}

El artículo que se publica es de exclusiva responsabilidad de los autores y no necesariamente reflejan el pensamiento de la Revista Ciencia Digital.

El artículo queda en propiedad de la revista y, por tanto, su publicación parcial y/o total en otro medio tiene que ser autorizado por el director de la Revista Ciencia Digital.
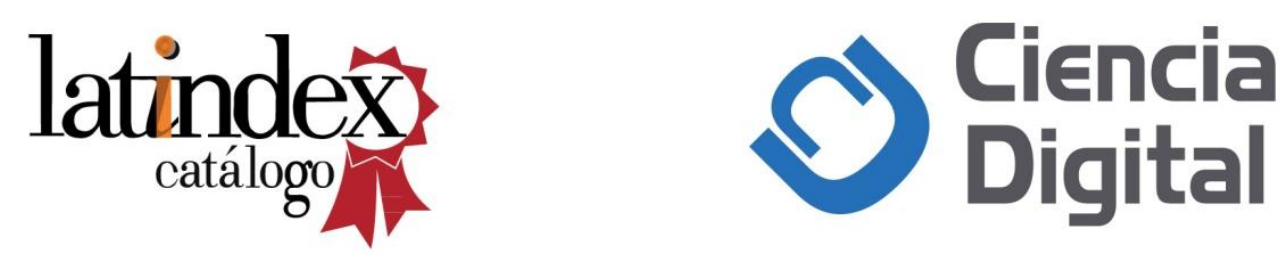